\title{
Cannabinoids in neurology - Brazilian Academy of Neurology
}

\author{
Canabinoides e seu uso em neurologia - Academia Brasileira de Neurologia \\ Sonia M. D. Brucki', Norberto Anísio Frota ${ }^{1}$, Pedro Schestatsky ${ }^{2}$, Adélia Henriques Souza ${ }^{3}$, Valentina Nicole \\ Carvalho ${ }^{3}$, Maria Luiza Giraldes Manreza ${ }^{3}$, Maria Fernanda Mendes4 ${ }^{4}$ Elizabeth Comini-Frota ${ }^{4}$, Cláudia \\ Vasconcelos ${ }^{4}$, Vitor Tumas ${ }^{5}$, Henrique B. Ferraz ${ }^{5}$, Egberto Barbosa ${ }^{5}$, Mauro Eduardo Jurno
}

\begin{abstract}
RESUMO
O uso do canabidiol em algumas condições neurológicas foi liberado pelo Conselho Regional de Medicina de São Paulo e pela Agência Nacional de Vigilância Sanitária (ANVISA). Especialistas em nome da Academia Brasileira de Neurologia prepararam uma posição crítica sobre o uso do canabidiol e outros derivados da cannabis em doenças neurológicas.
\end{abstract}

Palavras-chave: canabidiol, doenças neurológicas, esclerose múltipla, epilepsia, dor, doença de Parkinson.

\section{ABSTRACT}

The use of cannabidiol in some neurological conditions was allowed by Conselho Regional de Medicina de São Paulo and by Agência Nacional de Vigilância Sanitária (ANVISA). Specialists on behalf of Academia Brasileira de Neurologia prepared a critical statement about use of cannabidiol and other cannabis derivatives in neurological diseases.

Keywords: cannabidiol, neurological diseases, multiple sclerosis, epilepsy, pain, Parkinson's disease.

\section{ASPECTOS GERAIS}

Recentemente o uso de canabidiol foi liberado para prescrição aos médicos do Estado de São Paulo, pelo Cremesp (Conselho Regional de Medicina do Estado de São Paulo), em 9 de outubro de 2014. A ANVISA já liberou o seu uso medicinal por importação para vários casos; exige-se a prescrição e laudo médicos e termo de responsabilidade. Cada vez mais o uso terapêutico dos canabinoides têm sido discutidos. A Academia Brasileira de Neurologia através de seus Departamentos Científicos tomou sua posição de acordo com as evidências científicas sobre o uso dos canabinoides nas devidas doenças neurológicas.

Os canabinoides mais exuberantes são o $\Delta$ 9-tetrahidrocanabinol (THC) que possui propriedades psicoativas e o canabidiol (CBD), que não tem propriedades psicoativas. Existem no sistema nervoso central os endocanabinoides, sendo dois tipos mais abundantes: o 2-arachydonoyl glycerol e o n-arachidonoyl ethanolamide. Eles são liberados em resposta à atividade sináptica excitatória, sendo sintetizados no corpo e dendritos dos neurônios, em resposta ao aumento da concentração de cálcio intracelular. Inibem a liberação de neurotransmissores pela via final em terminais gabaérgicos e em menor extensão, glutamatérgicos. Agem em vários mecanismos de plasticidade de curto e longo prazo de sinapses inibitórias e excitatórias. Várias áreas cerebrais são ricas em receptores CB1, como o córtex frontal, os núcleos da base, cerebelo e na região límbica cerebral. Por estes mecanismos podem ter ação em várias doenças neurológicas ${ }^{1,2,3,4}$.

\section{EFEITOS COGNITIVOS}

O uso de cannabis na forma inalada, por indivíduos saudáveis, está associado a pior desempenho cognitivo, seja de forma aguda ou crônica. A suspensão do seu uso reverte parcialmente

\footnotetext{
${ }^{1}$ Academia Brasileira de Neurologia, DC Neurologia Cognitiva e do Envelhecimento; Sao Paulo SP, Brazil;

${ }^{2}$ Academia Brasileira de Neurologia, DC Dor; Sao Paulo SP, Brazil;

${ }^{3}$ Academia Brasileira de Neurologia, DC Epilepsia; Sao Paulo SP, Brazil;

${ }^{4}$ Academia Brasileira de Neurologia, DC Neuroimunologia; Sao Paulo SP, Brazil;

${ }^{5}$ Academia Brasileira de Neurologia, DC Distúrbios do Movimento; Sao Paulo SP, Brazil;

${ }^{6}$ Academia Brasileira de Neurologia, DC Cefaleia. Sao Paulo SP, Brazil.

Correspondence: Sonia MD Brucki; Rua Rio Grande, 180 / ap. 61; 04018-000 Sao Paulo SP, Brasil; E-mail: sbrucki@uol.com.br

Conflict of interest: There is no conflict of interest to declare.

Received 03 March 2015; Accepted 13 March 2015.
} 
esta queda, sem o normalizar. Poucos estudos avaliaram a influência na cognição do uso da cannabis na forma inalada em pacientes com doença neurológicas. Pacientes com esclerose múltipla que utilizaram cannabis de forma inalada, seja com intuito recreativo ou terapêutico, apresentaram pior desempenho cognitivo em teste de velocidade de processamento de informação, memória operacional, funções executivas e processamento visoespacial ${ }^{1}$. O uso de canabidiol parece não ter relação com declínio cognitivo, porém, poucos estudos avaliariam seu uso na população idosa ${ }^{5}$.

\section{CANABINOIDES EM EPILEPSIA}

O CBD tem reconhecido efeito antiepiléptico, porém, com mecanismo de ação, segurança a longo prazo, propriedades farmacocinéticas e interações com outros fármacos, ainda obscuros. As pesquisas clínicas bem conduzidas metodologicamente são limitadas, pois há restrição legal ao uso de medicamentos derivados do cannabis, embora o CBD não possua propriedades psicoativas.

Dr. Devinsky, na New York University School of Medicine foi autorizado pelo Federal Drug Administration (FDA) a conduzir um estudo aberto com um produto contendo $98 \%$ de CBD cujo nome comercial é Epidiolex fabricado pela GW Pharmaceuticals. A dose diária foi gradualmente aumentada até o máximo de $25 \mathrm{mg} / \mathrm{kg} /$ dia associada aos medicamentos que o paciente já utilizava. Os resultados dos primeiros 23 pacientes, cuja média de idade foi de 10 anos, demonstraram que $39 \%$ dos pacientes tiveram redução de $50 \%$ de suas crises. Obtiveram controle total das crises apenas 3 dos 9 pacientes com síndrome de Dravet (um tipo de epilepsia muito grave da infância) e 1 dos 14 pacientes com outras formas de epilepsia. Os efeitos colaterais mais comuns foram sonolência, fadiga, perda ou ganho de peso, diarreia e aumento ou redução do apetite. Todos os pacientes recebiam mais de um fármaco antiepiléptico. Os resultados preliminares mostraram redução de $50 \%$ de crises em cerca de $40 \%$ dos pacientes. Tal resultado não difere dos resultados disponíveis na literatura dos mais de 20 fármacos antiepilépticos disponíveis no mercado.

As populações expostas ao CBD são compostas por pacientes com síndromes epilépticas heterogêneas que não responderam a qualquer outro fármaco, ou tiveram sérios efeitos colaterais com os medicamentos disponíveis no mercado. Neste cenário, um composto que tenha qualquer efeito benéfico torna-se potencialmente útil.

Os dados científicos até agora disponíveis permitem concluir que o canabidiol poderá desempenhar um papel importante no tratamento de epilepsias muito difíceis, em casos específicos, ainda não definidos cientificamente.

Enfatizamos que o canabidiol terá aplicabilidade dentro do cenário das epilepsias intratáveis, de dificílimo controle, possivelmente com excelente resposta em alguns casos, razoável resposta em outros e nenhuma resposta em alguns, como observado com o uso de outros fármacos. A segurança e eficácia do CBD necessitam ser melhor estabelecidas por estudos bem conduzidos, uma vez que os dados disponíveis na literatura atual não preenchem os critérios científicos exigidos para que tal composto seja utilizado como medicamento de forma indiscriminada na epilepsia.

\section{CANABINOIDES NA ESCLEROSE MÚLTIPLA}

O uso da maconha na esclerose múltipla (EM) é frequentemente discutido no tratamento sintomático e preventivo. Alguns cuidados devem ser tomados quanto à indicação do uso de canabinoides na forma oral na EM, pois seus efeitos adversos podem ser agravados em função de características inerentes à doença. Sintomas como comprometimento cognitivo, fadiga e alterações humor, que podem variar de depressão à ideação suicida, devem ser avaliados antes da indicação destas substâncias na EM. O naxibimol é um preparado comercial, utilizado em alguns países com indicação específica para espasticidade na EM. Contém THC e CDB, na proporção de 1:1, de uso exclusivamente oro-bucal e utilizado na dose de máxima de até 12 puffs ao dia. Não existem estudos consistentes para indicação terapêutica da maconha na forma de cigarros em qualquer dos sintomas da EM. Existem estudos classe I, II e III para preparados orais e naxibimols para alguns dos sintomas da EM.

Para o tratamento da espasticidade: estudos com Naxibimols demonstraram melhora nas escalas de auto-avaliação em seis semanas, embora não fossem observadas melhoras nas escalas objetivas para espasticidade. Sua eficácia de longo prazo ainda não foi confirmada. O extrato de cannabis oral e o THC também se mostrou eficaz apenas nas escalas de auto-avaliação no uso por até 15 semanas, porém, após um ano os resultados indicaram uma melhora também nas escalas objetivas de mensuração da espasticidade. Estes resultados sugerem que esta opção terapêutica pode ser considerada nos pacientes com EM, embora faltem estudos de segurança com uso por longos períodos 6 .

Na dor neuropática ou central, os estudos foram realizados em períodos curtos, com eficácia variável. Os naxibimols, os preparados com THC/CBD e o extrato de cannabis apresentaram resultados conflitantes, e embora não seja possível concluir de forma definitiva quanto à sua eficácia, os dados sugerem que esta pode ser uma opção terapêutica em pacientes que não responderam aos tratamentos convencionais i,8,9,10,11. $^{7}$.

No tratamento dos tremores e da disfunção vesical, o uso dos naxibimols ou de preparados orais THC, CBD ou THC/CDB mostrou-se ineficaz, não havendo neste momento indicação para o seu uso no alívio deste sintoma.

Concluindo: o naxibimol pode ser utilizado na espasticidade e dor da EM, desde que esgotadas as demais 
possibilidades terapêuticas, sempre observando riscos e benefícios da sua indicação.

\section{CANABINOIDES NA DOENÇA DE PARKINSON E EM OUTROS DISTÚRBIOS DO MOVIMENTO}

A Academia Americana de Neurologia (AAN) publicou recentemente uma revisão sistemática sobre a eficácia e segurança do uso terapêutico da maconha e seus derivados no tratamento de doenças neurológicas ${ }^{10}$.

Desse extenso trabalho da AAN podemos verificar que há poucos estudos de qualidade disponíveis na literatura para termos uma conclusão final sobre o uso terapêutico dos derivados da cannabis em pacientes com distúrbios do movimento. Há que se considerar que o risco de efeitos psicopatológicos graves pode chegar a $1 \%$. Isso vai depender sem dúvida da proporção de THC presente no tratamento, mas de certa forma não há relato de efeitos colaterais graves. Os extratos de cannabis não melhoram as discinesias induzidas pela levodopa em pacientes com doença de Parkinson (DP).

Recentemente, estudos preliminares utilizando CBD puro no tratamento de pacientes portadores de DP revelaram um efeito positivo sobre os sintomas psicóticos, o sono e a qualidade de vida dos pacientes ${ }^{11}$. O CBD poderia ter um efeito terapêutico sobre os sintomas do transtorno comportamental do sono REM ${ }^{12}$.

Em conclusão, apesar da ausência de evidências suficientes para indicar o uso dos derivados da cannabis em pacientes com distúrbios do movimento, há sinais de que o uso de extratos da planta e especialmente de CBD possa ajudar a minimizar sintomas não-motores da DP como: psicose, distúrbios do sono, dor, talvez urgência miccional, e também promover uma melhora geral na qualidade de vida dos pacientes. O uso terapêutico sem indicação precisa só seria indicado em casos de distúrbios do movimento em que os tratamentos convencionais disponíveis falharam, e a qualidade de vida do paciente esteja muito comprometida. É provável que o uso de CBD puro e extratos de cannabis com baixo teor de THC sejam os mais eficientes e menos propensos a causar efeitos indesejáveis ${ }^{12}$.

\section{CANABINOIDES NO TRATAMENTO DE DOR NEUROPÁTICA}

Três estudos avaliaram a eficácia da marijuana no tratamento de dor neuropática. Em um deles foi utilizada a forma spray, como analgesia adjuvante no tratamento de dor central em pacientes com esclerose múltipla. Em outro estudo foi utilizada forma inalatória, em pacientes com dor neuropática pós-traumática ou pós-cirúrgica, com melhora na intensidade da dor ${ }^{13}$. Finalmente, Ellis et al. observaram melhora da dor neuropática em pacientes com HIV ${ }^{14}$.

Por se tratar de um tratamento do tipo SFBR (Simples, Fácil, Barato e Racional) em oposição a tratamentos dispendiosos, tóxicos e custosos, pode ser uma opção para casos de dor refratária, em falhas terapêuticas ou eficácia insuficiente. Para seu uso sistemático seria necessário maior volume de estudos ${ }^{15}$.

\section{CANABINOIDES NA CEFALEIA}

Não existem estudos recentes para seu uso na cefaleia. Apesar de algumas patologias relacionadas à dor do segmento cefálico responderem ao uso dos canabinoides, como na dor neuropática orofacial (neuralgia do trigêmeo, síndrome da boca ardente e dor orofacial persistente) e sua ação no sistema de dor central (sistema trigeminal e substância cinzenta periaquedutal) apresentarem intensa intersecção com as vias dolorosas envolvidas nas dores de cabeça, especialmente a migrânea, não podemos dizer, pela falta de estudos específicos que possa ser indicado para seu tratamento ${ }^{16,17,18}$.

\section{CONSIDERACุÕES FINAIS}

Parecem existir evidências de efeitos benéficos dos canabinoides em alterações do sistema nervoso central e periférico, porém, estudos de longo prazo devem ser realizados, com maior número de pacientes, com eficácia medida por instrumentos objetivos e seu uso a longo prazo ainda não é conhecido. O uso do canabidiol é indicado na falha terapêutica dos tratamentos já consagrados ou quando os mesmos apresentam eficácia insuficiente. $\mathrm{O}$ uso da cannabis de forma recreativa é contraindicada pela ABN.

\section{References}

\footnotetext{
1. Bennaroch EE. Synaptic effects of cannabinoids: complexity, behavioral effects and potential clinical implications. Neurology 2014;83:1958-67. http://dx.doi.org/10.1212/WNL.0000000000001013

2. Giacoppo S, Mandolino G, Galuppo M, Bramanti P, Mazzon E. Cannabinoids: new promising agents in the treatment of neurological diseases. Molecules 2014;19:18781-816. http://dx.doi.org/10.3390/molecules191118781
}

3. Mechoulam R, Hanuš LO, Pertwee R, Howlett AC. Early phytocannabinoid chemistry to endocannabinoids and beyond. Nat Rev Neurosci 2014;15:757-64. http://dx.doi.org/10.1038/nrn3811

4. Ahmed AIA, Elsen GAH, Colbers A, Marck MA, Burger DM, Feuth TB et al. Safety and pharmacokinetics of oral delta-9tetrahydrocannabinol in healthy older subjects: a randomized controlled trial. Eur Neuropsychopharmacol 2014;24:1475-82. http://dx.doi.org/10.1016/j.euroneuro.2014.06.007 
5. Elsen GAH, Ahmed AIA, Lamens M, Kramers C, Verkes RJ, Marck MA et al. Efficacy and safety of medical cannabinoids in older subjects: a systematic review. Ageing Res Rev. 2014;14: 56-64. http://dx.doi.org/10.1016/j.arr.2014.01.007

6. Notcutt W, Langford R, Davies P, Rarcliffe S, Potts R. A placebocontrolled, parallel-group, randomized withdrawal study of subjects with symptoms of spasticity due to multiple sclerosis who are receiving long-term Sativex (nabiximols). Mult Scler 2012; 18: 219-28. http://dx.doi.org/10.1177/1352458511419700

7. Ware MA, Wang T, Shapiro S, Robinson A, Ducruet T, Huynh T et al. Smoked cannabis for chronic neuropathic pain: a randomized controlled trial. CMAJ 2010;182:E694-701. http://dx.doi.org/10.1503/cmaj.091414

8. Honarmand K, Tierney MC, O'Connor P, Feinstein A. Effects of cannabis on cognitive function in patients with multiple sclerosis. Neurology 2011;769:1153-60. http://dx.doi.org/10.1212/WNL.0b013e318212ab0c

9. Zajicek JP, Hobart JC, Slade A, Barnes D, Mattison PG. Multiple sclerosis and extract of cannabis: results of MUSEC trial.J Neurol Neurosurg Psychiatr 2012;83:1125-32. http://dx.doi.org/10.1136/jnnp-2012-302468

10. Koppel BS, Brust JC, Fife T, Bronstein J, Youssof S, Gronseth G et al. Systematic review: efficacy and safety of medical marijuana in selected neurologic disorders: report of the Guideline Development Subcommittee of the American Academy of Neurology. Neurology. 2014;82: 1556-63. http://dx.doi.org/10.1212/WNL.0000000000000363

11. Chagas MH, Zuardi AW, Tumas V, Pena-Pereira MA, Sobreira ET, Bergamaschi MM et al. Effects of cannabidiol in the treatment of patients with Parkinson's disease: an exploratory double-blind trial.J Psychopharmacol 2014;28:1088-98. http://dx.doi.org/10.1177/0269881114550355
12. Chagas MH, Eckeli AL, Zuardi AW, Pena-Pereira MA, SobreiraNeto Ma, Sobreira ET et al. Cannabidiol can improve complex sleep-related behaviours associated with rapid eye movement sleep behaviour disorder in Parkinson's disease patients: a case series. J Clin Pharm Therapeutics 2014;39:564-6. http://dx.doi.org/10.1111/jcpt.12179

13. Ueda M, Iwasaki H, Wang S, Murata E, Poon KY, Mao J et al. Cannabinoid receptor type 1 antagonist, AM251, attenuates mechanical allodynia and thermal hyperalgesia after burn injury. Anesthesiology 2014;121:1311-9. http://dx.doi.org/10.1097/ALN.0000000000000422

14. Ellis RJ, Toperoff W, Vaida F, Brande G, Gonzales J, Gouaux B et al. Smoked medicinal cannabis for neuropathic pain in HIV:a randomized, crossover clinical trial. Neuropsychopharmacology 2009; 34:672-80. http://dx.doi.org/10.1038/npp.2008.120.

15. Schestatsky P, Vidor L, Winckler PB, Araújo TG, Caumo W. Promising treatments for neuropathic pain. Arq Neuropsiquiatr 2014; 72: 881-8. http://dx.doi.org/10.1590/0004-282X20140157.

16. McDonough P, McKenna JP, McCrary C, Downer EJ. Neuropathic orofacial pain: cannabinoids as a therapeutic avenue. Int J Biochem Cell Biol 2014; 55: 72-8. http://dx.doi.org/10.1016/j.biocel.2014.08.007.

17. Ueda M, Iwasaki H, Wang S, Murata E, Poon KY, Mao J et al. Cannabinoid receptor type 1 antagonist, AM251, attenuates mechanical allodynia and thermal hyperalgesia after burn injury. Anesthesiology 2014;121:1311-9. http://dx.doi.org/10.1097/ALN.0000000000000422.

18. Rog DJ, Nurmikko TJ, Friede T, Young CA. Randomized, controlled trial of cannabis-based medicine in central pain in multiple sclerosis. Neurology 2005;65:812-9. http://dx.doi.org/10.1212/01.wnl.0000176753.45410.8b 